\title{
A New and Efficient Boundary Element-Free Method for 2-D Crack Problems
}

\author{
Jinchao Yue, ${ }^{1}$ Liwu Chang, ${ }^{1,2}$ and Yuzhou Sun ${ }^{2}$ \\ ${ }^{1}$ School of Water Conservancy and Environment, Zhengzhou University, Zhengzhou 450001, China \\ ${ }^{2}$ School of Civil Engineering and Architecture, Zhongyuan University of Technology, Zhengzhou 450007, China
}

Correspondence should be addressed to Jinchao Yue; yuejc@zzu.edu.cn and Yuzhou Sun; yuzhousun@126.com

Received 4 September 2016; Revised 14 December 2016; Accepted 24 January 2017; Published 21 February 2017

Academic Editor: Elisa Francomano

Copyright (C) 2017 Jinchao Yue et al. This is an open access article distributed under the Creative Commons Attribution License, which permits unrestricted use, distribution, and reproduction in any medium, provided the original work is properly cited.

\begin{abstract}
An efficient boundary element-free method is established for 2-D crack problems by combining a pair of boundary integral equations and the moving-least square approximation. The displacement boundary integral equation is collated on the on-crack boundary, and a new traction boundary integral equation is applied on the crack surface without the separate consideration of the upper and lower sides. In virtue of integration by parts, only singularity in order $1 / r$ is involved in the integral kernels of new traction boundary integral equation, which brings convenience to the numerical implementation. Meanwhile, the integration by parts produces the new variables, the displacement density, and displacement dislocation density, and they are the coexisting unknowns along with the displacement and displacement dislocation. With the high-order continuity of the moving-least square approximation, these new variables are directly approximated with the nodal displacement or displacement dislocation, and the final system of equations contains the unknowns of nodal displacements and displacement dislocations only. The boundary element-free computational scheme is established, and several examples show the efficiency and flexibility of the proposed method.
\end{abstract}

\section{Introduction}

In comparison with the domain-type methods such as finite element method (FEM), the boundary integral equation (BIE) method show some special advantages for the fracture mechanics problems because it only requires the discretization of the general boundary and crack surface rather than the domain. But the traditional displacement BIE cannot directly be applied to crack bodies because the geometrical overlapping of the upper and lower crack surfaces leads to an indeterminacy of equations $[1,2]$. To overcome this difficulty, a direct way is to derive the traction BIE of cracked bodies [36], but the kernels of the traction BIE involve hypersingular terms and their evaluation is very arduous. A different approach is the dual BIE method, in which the displacement BIE is applied to the outside boundary and to one side of crack surface, and the traction BIE is applied to another side of crack surface [7-9]. To reduce the number of equations in the final system matrix, Pan and Amadei proposed a new pair of BIEs so that the displacement BIE is collocated only on the no-crack boundary, and the traction BIE is applied only on one side of crack surface [10,11]. But the evaluation of hypersingular integrals is still a vital task in the above methods. Actually, the hypersingular integral can be avoided by using integration by parts, and this skill has been used to derive a regular new traction BIE by Chau and Wang [12]. This skill has also been applied to the anisotropic medium and bimaterials by Sun et al. [13-15]. In this paper, we propose that an alternative computational scheme by combining the new traction BIE and displacement BIE, that is, the displacement $\mathrm{BIE}$, is collocated on the no-crack boundary, and the traction BIE only with the singular integral kernel in order of $1 / r$ is collocated along the entire crack surface (no need for the separate discretization of the upper and lower crack surfaces).

In the derivation of the new traction BIE, two new variables, the displacement density and displacement dislocation density, are introduced, and they are the basic coexisting unknowns with the displacement and displacement dislocation in the proposed computational scheme. So the new technique is needed to efficiently bridge these unknowns in the numerical simulation. Mesh-free/element-free methods 
emerge from the nonlocal interpolation/approximation techniques such as the moving-least square (MLS) approximation [16] that has no dependence on the elements. The domaintype element-free methods, such as element-free Galerkin method [17-19], can conveniently model the crack discontinuity with the enriched nodal degrees of freedom [20-22]. With the enlightenment of element-free methods, the extended FEM method has been proposed for the crack problems through the embedding of displacement jumps within elements [23-25]. BIE can also be combined with the nonlocal approximations to generate the boundary-type elementfree methods, for example, the called boundary node method $[26,27]$ and the called boundary element-free method [28$30]$, in which the unknowns are approximated with the MLS approximation [26], improving MLS approximation [28-30] or Shepard and Taylor interpolation [27]. Another advantage of element-free methods is that the shape function stratifies the higher-order continuity automatically, and the derivative of shape function can be constructed easily, which brings convenience to the higher-order continuity problems [3133]. For example, for the strain gradient structures, the strain gradient can be approximated directly with nodal displacement, and nodal displacements are the only unknowns in the final system matrix [33]. This advantage encourages us to establish a boundary element-free method to implement the numerical simulation, in which the displacement density and displacement dislocation density are directly approximated with the nodal displacement and displacement dislocation.

This paper will firstly present the new traction BIE only with singularity in order $1 / r$ by using integration by parts [12] and then combine it to the displacement BIE to construct a pair of BIEs for 2-D crack problems. The displacement BIE is collocated on the on-crack boundary, and the unknowns are the nodal displacements and displacement dislocations. The traction BIE is collocated on crack surface, and the unknown displacement density and displacement dislocation density are directly approximated with the nodal displacements and displacement dislocations, so that the final system of equations contains the nodal displacements and displacement dislocations as the only unknowns. Since the shape functions are digitally known in the present method and the singular integrals must be evaluated in a pure numerical scheme, the method of Torino [34] is employed to efficiently evaluate the Cauchy integrals.

\section{New Boundary Integral Equations}

Consider small displacement in two-dimensional, homogeneous, isotropic, and linear-elastic solids, the displacement, strain, and stress are, respectively, denoted as $u_{i}, \varepsilon_{i j}$, and $\sigma_{i j}$ $(i, j=1,2)$. The governing equations are given as

$$
\begin{aligned}
\varepsilon_{i k} & =\frac{1}{2}\left(u_{i, k}+u_{k, i}\right), \\
\sigma_{i j} & =\lambda \delta_{i j}\left(\varepsilon_{11}+\varepsilon_{22}\right)+2 G \varepsilon_{i j}, \\
\sigma_{i j, j}+b_{i} & =0,
\end{aligned}
$$

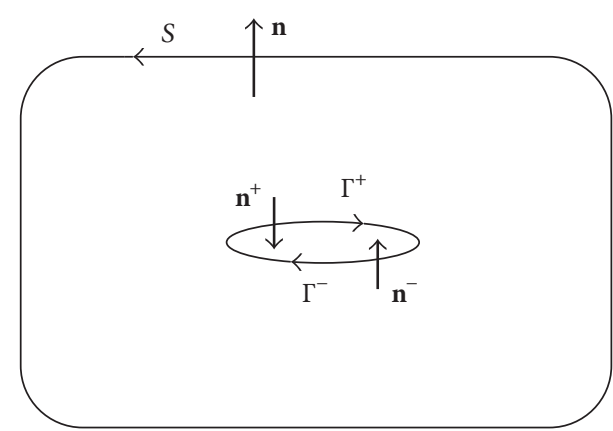

FIGURE 1: The positive direction and normal direction for the outside boundary $S$ and crack surface $\Gamma$.

where $b_{i}$ is the body force; $\lambda=G(3-\kappa) /(\kappa-1) ; G$ is the shear modulus; $\kappa$ equals $3-4 \nu$ for plane strain and $(3-v) /(1+\nu)$ for plane stress with $v$ being Poisson's ratio. Equation (2) can also be written as

$$
\begin{aligned}
\sigma_{i j} & =c_{i j k l} \varepsilon_{k l}, \\
c_{i j k l} & =\frac{2 G \nu}{1-2 \nu} \delta_{i j} \delta_{k l}+G\left(\delta_{i l} \delta_{j k}\right) .
\end{aligned}
$$

The fundamental solutions $U_{i j}\left(\mathbf{x}_{0}, \mathbf{x}\right)$ and $T_{i j}\left(\mathbf{x}_{0}, \mathbf{x}\right)$ are the $j$ th displacement and traction at the field point $\mathbf{x}\left(x_{1}, x_{2}\right)$ caused by a unit point force along the $i$ th direction at a source point $\mathbf{x}_{0}\left(x_{01}, x_{02}\right)$, and they can be expressed as follows $[1,2,36]$ :

$$
\begin{aligned}
& U_{i j}\left(\mathbf{x}_{0}, \mathbf{x}\right)=\frac{1}{2 \pi G(\kappa+1)}\left(\delta_{i j} \kappa \ln \frac{1}{r}+r_{, i} r_{, j}\right), \\
& T_{i j}\left(\mathbf{x}_{0}, \mathbf{x}\right)=-D_{i j k}\left(\mathbf{x}_{0}, \mathbf{x}\right) \cdot n_{k}(\mathbf{x}), \\
& D_{i j k}\left(\mathbf{x}_{0}, \mathbf{x}\right) \\
& \quad=\frac{1}{\pi(\kappa+1) r}\left[\frac{1}{2}(\kappa-1)\left(\delta_{i j} r_{, k}+\delta_{i k} r_{, j}-\delta_{j k} r_{, i}\right)\right. \\
& \left.\quad+2 r_{, i} r_{, j} r_{, k}\right],
\end{aligned}
$$

where $\delta_{i j}$ is the Kronecker delta; $r=\left|\mathbf{x}-\mathbf{x}_{0}\right|$ is the distance between points $\mathbf{x}$ and $\mathbf{x}_{0} ; n_{k}(\mathbf{x})$ is the unit normal along the boundary.

Consider a crack embedding in the elastic body as Figure 1, if its upper and lower surfaces are viewed as being geometrically overlapping, that is, $\mathbf{n}^{-}=-\mathbf{n}^{+}$, the Somigliana formula expresses the displacement at an interior point $\mathbf{x}_{0}\left(x_{01}, x_{02}\right)$ in terms of the traction $t_{j}(\mathbf{x})=\sigma_{i j} n_{i}$ and displacement $u_{j}(\mathbf{x})$ on the boundary point $\mathbf{x}\left(x_{1}, x_{2}\right)[1,12]$ :

$$
\begin{aligned}
u_{i}\left(\mathbf{x}_{0}\right)= & \int_{S} U_{i j}\left(\mathbf{x}_{0}, \mathbf{x}\right) t_{j}(\mathbf{x}) d s(\mathbf{x}) \\
& -\int_{S} T_{i j}\left(\mathbf{x}_{0}, \mathbf{x}\right) u_{j}(\mathbf{x}) d s(\mathbf{x})
\end{aligned}
$$




$$
\begin{aligned}
& +\int_{\Gamma} U_{i j}\left(\mathbf{x}_{0}, \mathbf{x}\right) \sum t_{j}(\mathbf{x}) d s(\mathbf{x}) \\
& -\int_{\Gamma} T_{i j}\left(\mathbf{x}_{0}, \mathbf{x}\right) \Delta u_{j}(\mathbf{x}) d s(\mathbf{x}),
\end{aligned}
$$

where $s(\mathbf{x})$ is the arc length along the boundary $S$ or crack surface $\Gamma ; \sum t_{j}(\mathbf{x})=t_{j}^{+}(\mathbf{x})+t_{j}^{-}(\mathbf{x})$ is the sum of the tractions acting on the upper and lower crack surfaces; $\Delta u_{j}(\mathbf{x})=$ $u_{j}^{+}(\mathbf{x})-u_{j}^{-}(\mathbf{x})$ is the difference of the displacements between the upper and lower crack surfaces.

Generally, $\sum t_{j}(\mathbf{x})$ in (6) is equal to zero, but it is not directly omitted at present, and this will lead to a different equation in comparison with the general traction boundary integral equation [3-5]. Let $\mathbf{x}_{0}$ trend to the no-crack boundary point; we obtain the following boundary integral equation:

$$
\begin{aligned}
\beta_{i j} u_{i}\left(\mathbf{x}_{0}\right)= & \int_{S} U_{i j}\left(\mathbf{x}_{0}, \mathbf{x}\right) t_{j}(\mathbf{x}) d s(\mathbf{x}) \\
& -\int_{S} T_{i j}\left(\mathbf{x}_{0}, \mathbf{x}\right) u_{j}(\mathbf{x}) d s(\mathbf{x}) \\
& +\int_{\Gamma} U_{i j}\left(\mathbf{x}_{0}, \mathbf{x}\right) \sum t_{j}(\mathbf{x}) d s(\mathbf{x}) \\
& -\int_{\Gamma} T_{i j}\left(\mathbf{x}_{0}, \mathbf{x}\right) \Delta u_{j}(\mathbf{x}) d s(\mathbf{x}),
\end{aligned}
$$

where $\beta_{i j}$ is the coefficient that depends only upon the local boundary geometry, and it is equal to $1 / 2$ for the smooth boundary.

It is well known, however, that for a cracked body, (7) does not have a unique solution because of the geometry singularity of the crack surface $[2,10,11]$. To overcome this difficulty, the traction integral equation is generally needed to be supplemented. In the dual BIE method [7-9], the displacement BIE is collocated on the no-crack boundary and on one side of crack surface while the traction integral equation is collocated on the other side of crack surface. To reduce the number of equations in the final system matrix, Pan and Amadei proposed a new pair of BIEs, so that the displacement integral equation is collocated only on the no-crack boundary, and a different traction integral equation is applied only on one side of the crack surface $[10,11]$. In the above methods, the integral kernels of traction BIE involve the hypersingular terms and their evaluation is very arduous. In the following, we will present a new traction integral equation [12] that involves the singular integral kernel only in order $1 / r$ and then combine it with (7) to solve the crack problems.

Since (6) is valid at all internal points of the linear-elastic body, we can differentiate $u_{i}\left(\mathbf{x}_{0}\right)$ with respect to $x_{0 k}$ to give

$$
\begin{aligned}
\frac{\partial u_{i}\left(\mathbf{x}_{0}\right)}{\partial x_{0 k}}= & \int_{S} \frac{\partial U_{i j}\left(\mathbf{x}_{0}, \mathbf{x}\right)}{\partial x_{0 k}} t_{j}(\mathbf{x}) d s(\mathbf{x}) \\
& -\int_{S} \frac{\partial T_{i j}\left(\mathbf{x}_{0}, \mathbf{x}\right)}{\partial x_{0 k}} u_{j}(\mathbf{x}) d s(\mathbf{x})
\end{aligned}
$$

$$
\begin{aligned}
& +\int_{\Gamma} \frac{\partial U_{i j}\left(\mathbf{x}_{0}, \mathbf{x}\right)}{\partial x_{0 k}} \sum t_{j}(\mathbf{x}) d s(\mathbf{x}) \\
& -\int_{\Gamma} \frac{\partial T_{i j}\left(\mathbf{x}_{0}, \mathbf{x}\right)}{\partial x_{0 k}} \Delta u_{j}(\mathbf{x}) d s(\mathbf{x}) .
\end{aligned}
$$

Using $\partial\left(x_{j}-x_{0 j}\right) / \partial x_{0 k}=-\partial\left(x_{j}-x_{0 j}\right) / \partial x_{k}$, we have

$$
\begin{aligned}
\frac{\partial U_{i j}\left(\mathbf{x}_{0}, \mathbf{x}\right)}{\partial x_{0 k}} & =-\frac{\partial U_{i j}\left(\mathbf{x}_{0}, \mathbf{x}\right)}{\partial x_{k}}=-U_{i j, k}\left(\mathbf{x}_{0}, \mathbf{x}\right), \\
\frac{\partial D_{i j k}\left(\mathbf{x}_{0}, \mathbf{x}\right)}{\partial x_{0 l}} & =-\frac{\partial D_{i j k}\left(\mathbf{x}_{0}, \mathbf{x}\right)}{\partial x_{l}} .
\end{aligned}
$$

Substituting (9) into (4), we have

$$
\begin{aligned}
c_{l m i k} \frac{\partial U_{i j}\left(\mathbf{x}_{0}, \mathbf{x}\right)}{\partial x_{0 k}} & =c_{l m i k} \frac{\partial U_{i j}\left(\mathbf{x}_{0}, \mathbf{x}\right)}{\partial x_{0 k}} \\
& =-c_{l m i k} \frac{\partial U_{j i}\left(\mathbf{x}_{0}, \mathbf{x}\right)}{\partial x_{k}}=D_{j l m}\left(\mathbf{x}_{0}, \mathbf{x}\right) ;
\end{aligned}
$$

here, $U_{i j}\left(\mathbf{x}_{0}, \mathbf{x}\right)=U_{j i}\left(\mathbf{x}_{0}, \mathbf{x}\right)$ is used.

The fundamental solution $D_{i j k}\left(\mathbf{x}_{0}, \mathbf{x}\right)$ should also satisfy the equilibrium equation, so we have the relationship

$$
\frac{\partial D_{i j 1}\left(\mathbf{x}_{0}, \mathbf{x}\right)}{\partial x_{1}}=-\frac{\partial D_{i j 2}\left(\mathbf{x}_{0}, \mathbf{x}\right)}{\partial x_{2}}, \text { for } \mathbf{x}_{0} \neq \mathbf{x}
$$

Furthermore, we can obtain

$$
\begin{aligned}
\frac{\partial T_{i j}\left(\mathbf{x}_{0}, \mathbf{x}\right)}{\partial x_{01}} & =-\left(n_{1} \frac{\partial D_{i j 1}\left(\mathbf{x}_{0}, \mathbf{x}\right)}{\partial x_{01}}+n_{2} \frac{\partial D_{i j 2}\left(\mathbf{x}_{0}, \mathbf{x}\right)}{\partial x_{01}}\right) \\
& =n_{1} \frac{\partial D_{i j 1}\left(\mathbf{x}_{0}, \mathbf{x}\right)}{\partial x_{1}}+n_{2} \frac{\partial D_{i j 2}\left(\mathbf{x}_{0}, \mathbf{x}\right)}{\partial x_{1}} \\
& =-\left(-n_{2} \frac{\partial}{\partial x_{1}}+n_{1} \frac{\partial}{\partial x_{2}}\right) D_{i j 2}\left(\mathbf{x}_{0}, \mathbf{x}\right) \\
& =-\frac{\partial D_{i j 2}\left(\mathbf{x}_{0}, \mathbf{x}\right)}{\partial s(\mathbf{x})} .
\end{aligned}
$$

The last step of (13) resulted from the identity $-n_{2}\left(\partial f / \partial x_{1}\right)+$ $n_{1}\left(\partial f / \partial x_{2}\right)=\partial f / \partial s$ (see Figure 1$)$

Similar procedure gives

$$
\frac{\partial T_{i j}\left(\mathbf{x}_{0}, \mathbf{x}\right)}{\partial x_{02}}=\frac{\partial D_{i j 1}\left(\mathbf{x}_{0}, \mathbf{x}\right)}{\partial s(\mathbf{x})}
$$


Substituting (8) into (2) or (4), using (11), (13), and (14), we have

$$
\begin{aligned}
\sigma_{l m}\left(\mathbf{x}_{0}\right)= & \int_{S} D_{j l m}\left(\mathbf{x}_{0}, \mathbf{x}\right) t_{j}(\mathbf{x}) d s(\mathbf{x}) \\
& +\int_{S} \frac{\partial W_{j l m}\left(\mathbf{x}_{0}, \mathbf{x}\right)}{\partial s(\mathbf{x})} u_{j}(\mathbf{x}) d s(\mathbf{x}) \\
& \cdot \int_{\Gamma} D_{j l m}\left(\mathbf{x}_{0}, \mathbf{x}\right) \sum t_{j}(\mathbf{x}) d s(\mathbf{x}) \\
& +\int_{\Gamma} \frac{\partial W_{j l m}\left(\mathbf{x}_{0}, \mathbf{x}\right)}{\partial s(\mathbf{x})} \Delta u_{j}(\mathbf{x}) d s(\mathbf{x})
\end{aligned}
$$

in which $W_{j l m}\left(\mathbf{x}_{0}, \mathbf{x}\right)=\lambda \delta_{l m} e_{k \beta} D_{l k \beta}\left(\mathbf{x}_{0}, \mathbf{x}\right)+G\left[e_{m \beta} D_{l j \beta}\left(\mathbf{x}_{0}, \mathbf{x}\right)\right.$ $\left.+e_{l \beta} D_{m j \beta}\left(\mathbf{x}_{0}, \mathbf{x}\right)\right]$ and $e_{i j}$ is $2-\mathrm{D}$ permutation tensor; that is, $e_{11}=e_{22}=0$ and $e_{12}=-e_{21}=1$.

Applying integration by parts to the second and fourth integrals of (15) and then letting $\mathbf{x}_{0}$ tend to the crack upper or lower surface, the limit of $n_{m}\left(\mathbf{x}_{0}\right) \sigma_{l m}\left(\mathbf{x}_{0}\right)$ gives

$$
\begin{aligned}
\beta_{l m}( & \left.t_{l}\left(\mathbf{x}_{0}^{+}\right)-t_{l}\left(\mathbf{x}_{0}^{-}\right)\right) \\
= & n_{m}\left(\mathbf{x}_{0}\right) \int_{s} D_{j l m}\left(\mathbf{x}_{0}, \mathbf{x}\right) t_{j}(\mathbf{x}) d s(\mathbf{x}) \\
& -n_{m}\left(\mathbf{x}_{0}\right) \int_{s} W_{j l m}\left(\mathbf{x}_{0}, \mathbf{x}\right) \frac{\partial u_{j}(\mathbf{x})}{\partial s(\mathbf{x})} d s(\mathbf{x}) \\
& +n_{m}\left(\mathbf{x}_{0}\right) \int_{\Gamma} D_{j l m}\left(\mathbf{x}_{0}, \mathbf{x}\right) \sum t_{j}(\mathbf{x}) d s(\mathbf{x}) \\
& -n_{m}\left(\mathbf{x}_{0}\right) \int_{\Gamma} W_{j l m}\left(\mathbf{x}_{0}, \mathbf{x}\right) \frac{\partial \Delta u_{j}(\mathbf{x})}{\partial s(\mathbf{x})} d s(\mathbf{x}),
\end{aligned}
$$

where $\beta_{l m}$ is the coefficient similar to the one in (7) and $t_{l}\left(\mathbf{x}_{0}^{+}\right)-t_{l}\left(\mathbf{x}_{0}^{-}\right)$is the difference of the tractions between the upper and lower crack surfaces.

Equation (16) does not involve the hypersingular integral kernels, and it is very helpful for the numerical computation. On the other hand, the integration by parts produces the new variables $\partial u_{j} / \partial s$ and $\partial \Delta u_{j} / \partial s$ with the physical meaning: the displacement densities and dislocation densities. The crack boundary conditions on the upper and lower surface have been incorporated into (16), so there is no need to discretize the upper and lower crack surfaces separately in the numerical implementation. Although, $\sum t_{j}(x)$ in (6) is generally equal to zero, the traction difference $t_{l}\left(z_{0}^{+}\right)-t_{l}\left(z_{0}^{-}\right)$may not be zero and may need to be considered, so it is vital to not directly omit $\sum t_{j}(x)$ in (6). In the following boundary element-free method, the displacement BIE (7) is applied to oncrack boundary, and (16) is applied to the crack surface.

\section{The MLS Approximation for the Unknowns}

The MLS approximation is a flexible nonlocal interpolation technique. Particularly, the MLS approximation can produce the higher-order continuity shape function automatically, and the derivative of unknowns can directly be approximated with the nodal parameters. While (7) is used, the following approximations are used:

$$
\begin{gathered}
u_{j}(\mathbf{x})=\sum_{I=1}^{N} \phi_{I} u_{j I}, \\
t_{j}(\mathbf{x})=\sum_{I=1}^{N} \phi_{I} t_{j I},
\end{gathered}
$$

where $u_{j I}$ and $t_{j I}$ are the nodal parameters; $\phi_{I}(\mathbf{x})$ is the MLS shape function; $N$ is the number of nodes which are covered by the effect domain of the evaluated point.

While (16) is used, the displacement density on the oncrack boundary is approximated as

$$
\frac{\partial u_{j}(\mathbf{x})}{\partial s(\mathbf{x})}=\sum_{I=1}^{N} \phi_{I, s} u_{j I}
$$

in which $\phi_{I, s}(\mathbf{x})$ is the derivative of the MLS shape function.

Referring to previous researches $[13,15]$, the crack displacement dislocation can be approximated using

$$
\Delta u_{j}=\sqrt{\left(\frac{s_{1}-s_{2}}{2}\right)^{2}-\left(s-\frac{s_{1}+s_{2}}{2}\right)^{2}} \sum_{I=1}^{N} \phi_{I} F_{j I} \text {, }
$$

where $s_{1}$ and $s_{2}$ are the arc coordinates of two crack tips and $F_{j I}$ is the nodal parameter. The displacement dislocation density can thus be approximated as

$$
\begin{aligned}
& \frac{\partial \Delta u_{j}}{\partial s}(s) \\
& =-\frac{1}{\sqrt{\left(\left(s_{1}-s_{2}\right) / 2\right)^{2}-\left(s-\left(s_{1}+s_{2}\right) / 2\right)^{2}}} \sum_{I=1}^{N} \phi_{I} F_{j I} \\
& \quad+\sqrt{\left(\left(s_{1}-s_{2}\right) / 2\right)^{2}-\left(s-\left(s_{1}+s_{2}\right) / 2\right)^{2}} \sum_{I=1}^{N} \phi_{I, S} F_{j I} .
\end{aligned}
$$

The MLS approximation is summarily described here, and the related details can be learned from the related literatures [16-18]. In MLS approximation, the trail function is written as

$$
F^{h}(s)=\sum_{i=1}^{m} p_{i}(s) a_{i}(s)=\mathbf{p}^{T}(s) \mathbf{a}(s),
$$

where $p_{i}(s), i=1,2, \ldots, m$ are monomial basis functions; $m$ is the number of terms in the basis; and $a_{i}(s)$ are coefficients of the basis functions. Examples of commonly used bases are the linear basis

$$
\mathbf{p}^{T}=(1, s)
$$

and the quadratic basis

$$
\mathbf{p}^{T}=\left(1, s, s^{2}\right)
$$


Unknown coefficients $a_{i}(s)$ in (21) can be determined by minimizing the weighted discrete $L_{2}$ norm

$$
J=\sum_{I}^{N} w\left(s-s_{I}\right)\left[\mathbf{P}^{T}\left(s_{\mathbf{I}}\right) \mathbf{a}\left(s_{\mathbf{I}}\right)-F_{\mathbf{I}}\right]^{2}
$$

where $w\left(s-s_{I}\right)$ is the weight function with compact support; $N$ is the number of nodes with $w\left(s-s_{I}\right)>0$; and $F_{\mathrm{I}}$ is the nodal parameter. The minimum of $J$ in (24) with respect to $\mathbf{a}(s)$ leads to a set of linear equations

$$
\mathbf{A}(s) \mathbf{a}(s)=\mathbf{B}(s) \mathbf{u},
$$

where

$$
\begin{gathered}
\mathbf{A}(s)=\sum_{\mathbf{I}}^{\mathbf{N}} w\left(s-s_{\mathrm{I}}\right) \mathbf{p}\left(s_{\mathrm{I}}\right) \mathbf{p}^{\mathrm{T}}\left(s_{\mathrm{I}}\right) \\
\mathbf{B}(s)=\left[w\left(s-s_{1}\right) \mathbf{p}\left(s_{1}\right), w\left(s-s_{2}\right)\right. \\
\left.\quad \cdot \mathbf{p}\left(s_{2}\right), \ldots, w\left(s-s_{N}\right) \mathbf{p}\left(s_{N}\right)\right]
\end{gathered}
$$

Thus, unknown coefficients a $(s)$ can be obtained from (25) as

$$
\mathbf{a}(s)=\mathbf{A}^{-1}(s) \mathbf{B}(s) \mathbf{u} .
$$

Substituting (27) into (21), the MLS approximation can be written in a standard form as

$$
F^{h}(s)=\sum_{\mathbf{I}=\mathbf{1}}^{\mathbf{N}} \phi_{\mathbf{I}}(s) F_{\mathbf{I}}(s)
$$

where the MLS shape function $\phi_{\mathbf{I}}(s)$ is defined as

$$
\begin{aligned}
\phi_{\mathbf{I}}(s) & =\sum_{i=1}^{m} p_{i}(s)\left[\mathbf{A}^{-1}(s) \mathbf{B}(s)\right]_{i \mathbf{I}} \\
& =\mathbf{p}^{T}(s) \mathbf{A}^{-1}(s) \mathbf{B}_{\mathbf{I}}(s)
\end{aligned}
$$

with

$$
\mathbf{B}_{\mathbf{I}}(s)=w\left(s-s_{\mathbf{I}}\right) \mathbf{p}\left(s_{\mathbf{I}}\right) .
$$

The derivatives of shape function can be obtained as

$$
\phi_{\mathbf{I}, s}(s)=\sum_{j=1}^{m}\left[p_{j, s}\left(\mathbf{A}^{-1} \mathbf{B}\right)_{j \mathbf{I}}+p_{j}\left(\mathbf{A}^{-1} \mathbf{B}_{, s}+\mathbf{A}_{, s}^{-1} \mathbf{B}\right)_{j \mathbf{I}}\right]
$$

in which

$$
\begin{aligned}
& \mathbf{B}_{, s}(s) \\
& =\left[w_{1, s}(s) \mathbf{p}\left(s_{1}\right), w_{2, s}(s) \mathbf{p}\left(s_{2}\right), \ldots, w_{m, s}(s) \mathbf{p}\left(s_{m}\right)\right] \\
& \mathbf{A}_{, s}^{-1}=-\mathbf{A}^{-1} \mathbf{A}_{, s} \mathbf{A}^{-1} \\
& A_{, s}(s)=\sum_{I=1}^{m} w_{I, s}(s) \mathbf{g}\left(s_{I}\right) \mathbf{p}^{T}\left(s_{I}\right) .
\end{aligned}
$$

In the present work, the quadratic basis is used, and the weight function is chosen as [16]

$$
w(d)= \begin{cases}\frac{2}{3}-4 d^{2}+4 d^{3}, & d \leq \frac{1}{2} \\ \frac{4}{3}+4 d+4 d^{2}-\frac{4}{3} d^{3}, & \frac{1}{2}<d \leq 1 \\ 0, & d>1 .\end{cases}
$$

The compact support domain of the evaluation point is determined by choosing the suitable quantity $R_{0}$ in the formula

$$
d=\frac{\left|s_{i}-s_{0 i}\right|}{R_{0}}, \quad(i=1,2) .
$$

\section{Boundary Element-Free Method}

To carry out the numerical integration, the outside boundary and crack surface are firstly separated into a series of subdomains that are also called "integral cells" [16-18]. Some nodes are then selected on each cell. The choice of cells is arbitrary and independent on nodes, but each cell must contain enough nodes to ensure that all compact supports domains cover the total boundary.

In this paper, a crack is uniformly collocated $M$ nodes and is simultaneously separated into $M$ integral cells uniformly. For the outside boundary, the integral cells are arranged in accordance with the nodal collocation; that is, two adjacent nodes denote an integral cell. The source point is chosen as the midpoint of integral cells in turn after the substitution of (17)-(20) into (7) and (16), and the numerical integrals are performed on every cell, which gives a series of linear equations. The solution of linear equations gives the nodal parameters, and the nodal displacements, displacement dislocations, displacement densities, and displacement dislocation densities can be evaluated with (17)-(20).

The integral cells are only used so that the integral can be evaluated numerically. The cell is not a boundary element, and the shape function is independent of it. The present method is called the boundary element-free method.

Consider the expressions of $U_{i j}\left(\mathbf{x}_{0}, \mathbf{x}\right), T_{i j}\left(\mathbf{x}_{0}, \mathbf{x}\right)$, $D_{j l m}\left(\mathbf{x}_{0}, \mathbf{x}\right)$, and $W_{j l m}\left(\mathbf{x}_{0}, \mathbf{x}\right)$, when the source point is located inside an integral cell that does not contain any crack tip; we need to evaluate the singular integrals in the forms

$$
\begin{gathered}
\int_{0}^{b} f(r) \ln \frac{1}{r} d r \\
\int_{a}^{b} \frac{f(r)}{|r|} d r \quad(a<0, b>0),
\end{gathered}
$$

where $f(r)$ is the smooth function on the integral cells.

If the integral cell includes a crack tip at its left or right end, we need to evaluate the singular integrals in the forms

$$
\begin{gathered}
\int_{0}^{b} \frac{f(r)}{\sqrt{s_{e} / 2-r}} \ln \frac{1}{r} d r, \\
\int_{a}^{b} \frac{f(r)}{|r| \sqrt{s_{e} / 2-r}} d r \quad(a<0, b>0),
\end{gathered}
$$




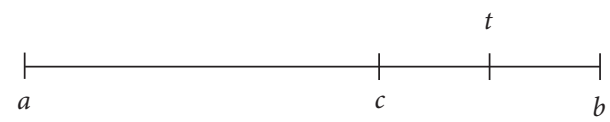

FIGURE 2: The integral interval is divided into two segments for the evaluation of the singular integrals.

where $s_{e}$ is the length of the integral cells that include the crack tip and $f(r)$ is the smooth function on the integral cells. Two integrals in (38) show the weak singularity $1 / \sqrt{s_{e} / 2-r}$ at the crack tip. By using a simple transformation $t=\sqrt{s_{e} / 2-r}$, this weak singularity can be eliminated and these two integrals can be transformed as the integrals in forms of (37); that is, we need to find the appropriate approaches to evaluate the logarithmic singularity integral $\int_{0}^{b} f(r) \ln (1 / r) d r$ and Cauchy singularity integral $\int_{a}^{b}(f(r) /|r|) d r(a<0, b>0)$.

Since the shape functions are digitally known in the present method, the singular integrals must be evaluated in a pure numerical scheme. The logarithmic singularity integral can be numerically evaluated using Gauss quadrature rule of logarithmic functions [37]. For the Cauchy singular integral, we find the method of Torino [34] to be very effective, and it has been used in our previous researches $[13,38]$. This method is simply described as follows.

As shown in Figure 2, the singular pole $t$ is located on the integral interval from $a$ to $b$ (assuming that point $t$ is nearer to $b$ than to $a$ ). A suitable point $c$ is selected so that point $t$ is equidistant from $c$ and $b .2 m$ point Gauss ruler points are used to draw the segments from $a$ to $c$ and from $c$ to $b$, and the total integral is then evaluated with the degree of exactness $4 m$ as

$$
\begin{aligned}
\mathbf{P} \int_{a}^{b} \frac{f(x)}{x-t} d x= & \int_{a}^{c} \frac{f(x)}{x-t} d t+\mathbf{P} \int_{c}^{b} \frac{f(x)}{x-t} d x \\
= & A_{0}^{(1)} f(t) \\
& +\sum_{i=1}^{2 m}\left[A_{i}^{(1)} f\left(x_{i}\right)+A_{i}^{(2)} f\left(y_{i}\right)\right] \\
& +R_{(2 m)}^{(2)}(f),
\end{aligned}
$$

where

$$
\begin{aligned}
x_{i} & =\frac{(c-a) \xi_{i}}{2}+\frac{a+c}{2}, \\
y_{i} & =\frac{(b-c) \xi_{i}}{2}+\frac{b+c}{2}, \\
A_{i}^{(1)} & =\frac{(c-a) H_{i}}{2\left(x_{i}-t\right)}, \\
A_{i}^{(2)} & =\frac{(b-c) H_{i}}{2\left(y_{i}-t\right)}, \\
A_{0}^{(1)} & =\ln \left|\frac{c-t}{a-t}\right|-\sum_{i=1}^{2 m} A_{i}^{(1)}
\end{aligned}
$$

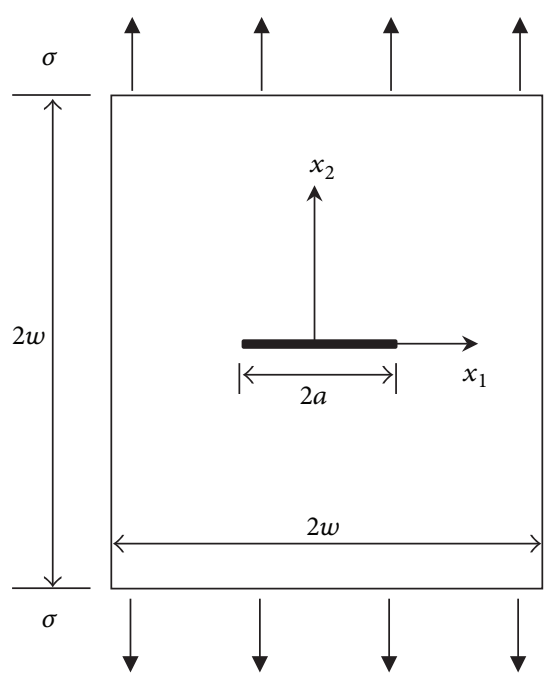

FIGURE 3: A horizontal straight crack in a square elastic plate.

with $\xi_{i}$ and $H_{i}$ being the standard Gauss quadrature point and weight, respectively.

Around a crack tip, the relation between the crack opening displacements and the extended stress intensity factors is $[11,13]$

$$
\begin{gathered}
K_{I}=\lim _{\rho \rightarrow 0}\left[\frac{G}{\kappa+1} \sqrt{\frac{2 \pi}{\rho}} u_{2}\right], \\
K_{I I}=\lim _{\rho \rightarrow 0}\left[\frac{G}{\kappa+1} \sqrt{\frac{2 \pi}{\rho}} u_{1}\right],
\end{gathered}
$$

where $\rho$ is the distance ahead of the crack tip. So $K_{I}$ and $K_{I I}$ can be evaluated with $F_{1}$ and $F_{2}$ corresponding to the crack tip nodes based on (19) and (20).

\section{Numerical Examples}

Four numerical examples are presented in this section. The first one is to verify the convergence and stability, and the other examples are to check the efficiency and advantages of the present method. During the numerical simulations, the scale $R_{0}$ is fixed as two times the distance between the adjacent nodes to ensure that four nodes are involved for the ordinary evaluation points.

5.1. Straight Crack in a Square Plate. The first example is a square plate that contains a horizontal straight crack along the $x_{1}$-axis (Figure 3 ). The length of the crack is $2 a$, and the origin of the Cartesian coordinate system $o x_{1} x_{2}$ is located at the crack center. The plate is under a uniform tensile stress $\sigma$ along $x_{2}$. The plate dimension is fixed as $w / a=2.0$. The computation indicates that good results can be obtained while 80 nodes are used on the outside boundary. Table 1 gives normalized stress intensity factors $Y_{I}=K_{I} / \sigma \sqrt{\pi a}$ for various numbers of nodes collocated on crack surface. It can be seen that a stable convergence can be achieved, and the error is 
TABLE 1: The normalized stress intensity factors $Y_{I}=K_{I} / \sigma \sqrt{\pi a}$ for a square plate.

\begin{tabular}{lccccccc}
\hline Node number & 5 & 10 & 15 & 20 & 25 & 30 & Ref. [35] \\
\hline$Y_{I}$ & 1.2921 & 1.3209 & 1.3292 & 1.3296 & 1.3298 & 1.3296 & 1.334 \\
\hline
\end{tabular}

TABLE 2: The normalized stress intensity factors for the crack tips $A$.

\begin{tabular}{lcccc}
\hline \multirow{2}{*}{$b / a$} & \multicolumn{2}{c}{$K_{I A} / \sigma \sqrt{\pi c}$} & \multicolumn{2}{c}{$K_{I I A} / \sigma \sqrt{\pi c}$} \\
& Present & Ref [8] & Present & Ref [8] \\
\hline 0.2 & 0.9941 & 0.993 & 0.0298 & 0.030 \\
0.4 & 0.9897 & 0.989 & 0.0352 & 0.036 \\
0.6 & 0.9873 & 0.987 & 0.0306 & 0.032 \\
\hline
\end{tabular}

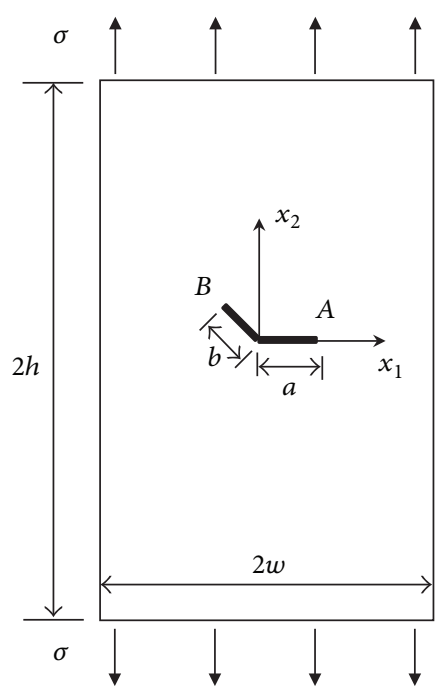

Figure 4: An internal kinked crack in a rectangular plate.

TABLE 3: The normalized stress intensity factors for the crack tips $B$.

\begin{tabular}{lcccc}
\hline \multirow{2}{*}{$b / a$} & \multicolumn{2}{c}{$K_{I B} / \sigma \sqrt{\pi c}$} & \multicolumn{2}{c}{$K_{I I B} / \sigma \sqrt{\pi c}$} \\
& Present & Ref [8] & Present & Ref [8] \\
\hline 0.2 & 0.5955 & 0.604 & 0.5613 & 0.556 \\
0.4 & 0.5683 & 0.576 & 0.6107 & 0.603 \\
0.6 & 0.5669 & 0.570 & 0.6296 & 0.624 \\
\hline
\end{tabular}

just $0.3 \%$ in comparison with [35] while 20 nodes are used for crack surface.

5.2. A Kinked Crack in a Rectangular Plate. Now, consider a rectangular plate with an internal kinked crack (Figure 4). The horizontal segment has the length $a$, and the other segment has the length $b$ and makes an angle of $45^{\circ}$ with the horizontal one. The horizontal projection of the total crack is $c=a+b / \sqrt{2}$. The kink of crack is at the center of plate, which has a height equal to twice the width; that is, $h=2 w$. The plate is under a uniform tensile stress $\sigma$ along $x_{2} . a / w$ is fixed as 0.1 , and $b / a$ is taken as $0.2,0.4$, and 0.6 . The normalized stress intensity factors are defined as $K_{I} / \sigma \sqrt{\pi c}$ and $K_{I I} / \sigma \sqrt{\pi c} .120$ nodes are used on the outside boundary, and 30 nodes are

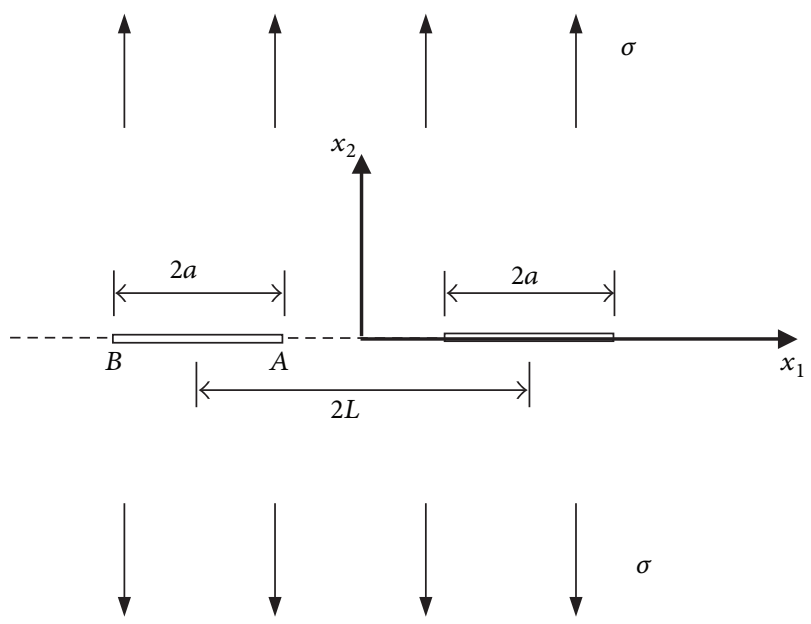

FIGURE 5: Two collinear cracks in an infinite elastic plate subjected to the remote uniform tension stress.

used for the horizontal segment and 6, 12, or 18 nodes are used, respectively, for the inclined segment in the cases of $b / a$ $=0.2,0.4$, or 0.6 . The stress intensity factors for crack tips $A$ and $B$ are listed in Tables 2 and 3. The results show a good agreement with those in [8].

5.3. Two Collinear Cracks in an Infinite Plate. This example studies the interaction between two collinear cracks with the same length in an infinite elastic plate (Figure 5), and the plate is subjected to the uniform remote tension $\sigma$ along the $x_{2}$ direction. The crack length is $2 a$, and the distance between two crack centers is $2 L$. When the distance between the centers of two cracks is $L=2 a$, the obtained normalized stress intensity factors are as follows: $Y_{I A}=K_{I A} / \sigma \sqrt{\pi a}=$ 1.0259 and $Y_{I B}=K_{I B} / \sigma \sqrt{\pi a}=1.0466$ (20 integral cells are uniformly collocated on each crack surface), and these results are very close to the reported results [35]. Figure 6 plots $Y_{I A}$ and $Y_{I B}$ versus $a / L$, which indicates that the interaction between two collinear cracks is considerably large only when they are very close to each other.

5.4. Circular-Arc Crack in an Unbounded Domain. To demonstrate the validity of the present method for the curved crack, a circular-arc crack in an infinite elastic medium is considered (Figure 7). The crack region is under uniform tension $\sigma$ in two perpendicular directions. The normalized stress intensity factors are denoted as $Y_{I, I I}=K_{I, I I} / \sigma \sqrt{\pi R \sin (\varphi)}$, and they are plotted as a function of the semiangle $\varphi$ in Figure 7. The present results are very close to the report results [35], and it is shown that the method is very effective for the curved crack since the equation is built on the arc coordinate system. 


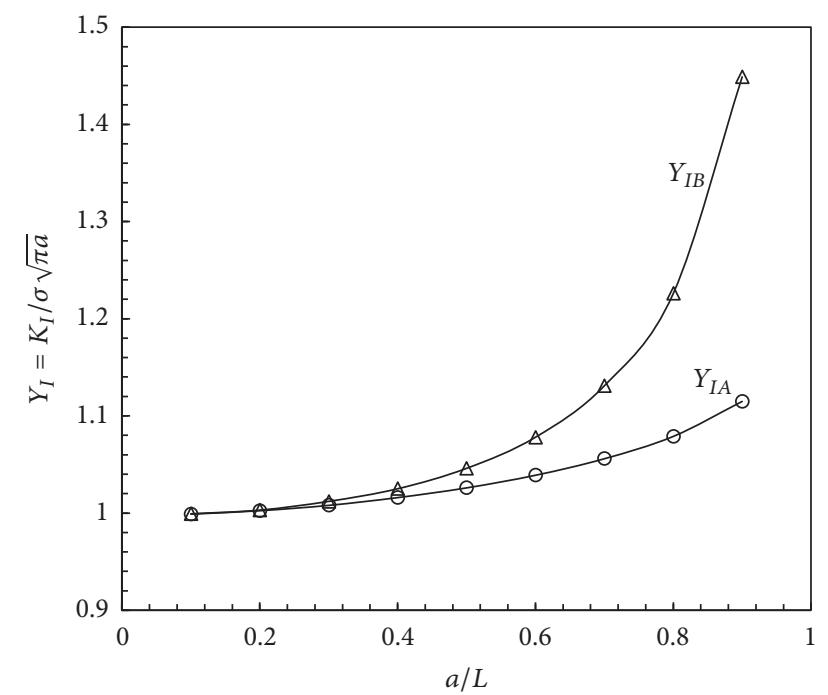

FIGURE 6: The normalized mode $I$ stress intensity factors versus $a / L$.

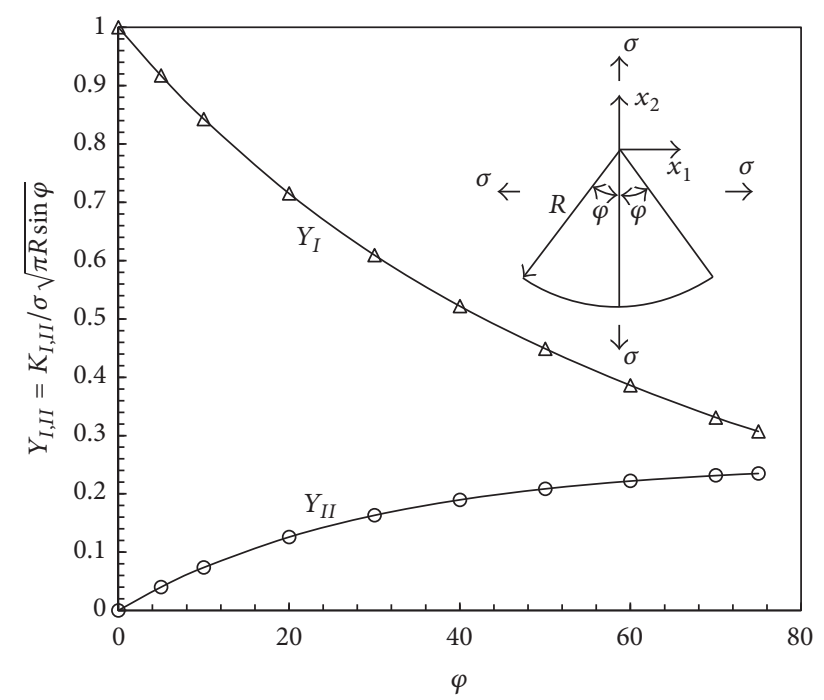

FIgURE 7: The normalized modes $I$ and $I I$ stress intensity factors versus a circular-arc crack.

\section{Conclusions}

The integration by parts is used to obtain a new traction BIE in which the hypersingularity integral is avoided, and it is combined with the displacement BIE to construct a pair of BIEs for the crack problems. The proposed method brings convenience to the numerical implementation. The negative consequence of integration by parts is the introduction of two new variables, the displacement density and displacement dislocation density, and they are the coexisting unknowns along with the displacement and displacement dislocation. In virtue of the high-order continuity of the MLS approximation, we propose to approximate the new variables directly with the nodal displacement and displacement dislocation, so that nodal displacement density and displacement dislocation density are not contained in the system of equations of the established element-free method. The method is proved to be very efficient for the general crack problems, and it is also shown that the MLS approximation can be well used in the problems that have the higher-order continuity requirement.

\section{Competing Interests}

The authors declare that there is no conflict of interests regarding the publication of this paper.

\section{Acknowledgments}

This work was supported by National Natural Science Foundation of China [Grant no. 11472316], Plan for Scientific Innovation Talent of Henan Province, China [Grant no. 164200510020], and Natural Science Foundations from Department of Education of Henan Province, China [Grant nos. 13B560304 and 15A560045].

\section{References}

[1] T. A. Cruse, Boundary Element Analysis in Computational Fracture Mechanics, vol. 1 of Mechanics: Computational Mechanics, Kluwer Academic, Dordrecht, Netherlands, 1988.

[2] M. H. Aliabadi, "Boundary element formulations in fracture mechanics," Applied Mechanics Reviews, vol. 50, no. 2, pp. 8396, 1997.

[3] T. Y. Qin and R. J. Tang, "Finite-part integral and boundary element method to solve embedded planar crack problems," International Journal of Fracture, vol. 60, no. 4, pp. 373-381, 1993.

[4] T. Rangelov, P. Dineva, and D. Gross, "A hyper-singular traction boundary integral equation method for stress intensity factor computation in a finite cracked body," Engineering Analysis with Boundary Elements, vol. 27, no. 1, pp. 9-21, 2003.

[5] Y. X. Mukherjee, K. Shah, and S. Mukherjee, "Thermoelastic fracture mechanics with regularized hypersingular boundary integral equations," Engineering Analysis with Boundary Elements, vol. 23, no. 1, pp. 89-96, 1999.

[6] G. Xie, J. Zhang, C. Huang, C. Lu, and G. Li, "A direct traction boundary integral equation method for three-dimension crack problems in infinite and finite domains," Computational Mechanics, vol. 53, no. 4, pp. 575-586, 2014.

[7] H.-K. Hong and J.-T. Chen, "Derivations of integral equations of elasticity," Journal of Engineering Mechanics, vol. 114, no. 6, pp. 1028-1044, 1988.

[8] A. Portela, M. H. Aliabadi, and D. P. Rooke, "The dual boundary element method: effective implementation for crack problems," International Journal for Numerical Methods in Engineering, vol. 33, no. 6, pp. 1269-1287, 1992.

[9] I. A. Alatawi and J. Trevelyan, "A direct evaluation of stress intensity factors using the extended dual boundary element method," Engineering Analysis with Boundary Elements, vol. 52, pp. 56-63, 2015.

[10] E. Pan and B. Amadei, "Fracture mechanics analysis of cracked 2-D anisotropic media with a new formulation of the boundary element method," International Journal of Fracture, vol. 77, no. 2, pp. 161-174, 1996.

[11] E. Pan, "A general boundary element analysis of 2-D linear elastic fracture mechanics," International Journal of Fracture, vol. 88, no. 1, pp. 41-59, 1997. 
[12] K. T. Chau and Y. B. Wang, "A new boundary integral formulation for plane elastic bodies containing cracks and holes," International Journal of Solids and Structures, vol. 36, no. 14, pp. 20412074, 1999.

[13] Y. Sun, Z. Zhang, S. Kitipornchai, and K. M. Liew, "Analyzing the interaction between collinear interfacial cracks by an efficient boundary element-free method," International Journal of Engineering Science, vol. 44, no. 1-2, pp. 37-48, 2006.

[14] Y.-Z. Sun, S.-S. Yang, and Y.-B. Wang, "A new formulation of boundary element method for cracked anisotropic bodies under anti-plane shear," Computer Methods in Applied Mechanics and Engineering, vol. 192, no. 22-23, pp. 2633-2648, 2003.

[15] Y. Sun and K. M. Liew, "Analyzing interaction between coplanar square cracks using an efficient boundary element-free method," International Journal for Numerical Methods in Engineering, vol. 91, no. 11, pp. 1184-1198, 2012.

[16] T. Belytschko, Y. Krongauz, D. Organ, M. Fleming, and P. Krysl, "Meshless methods: an overview and recent developments," Computer Methods in Applied Mechanics and Engineering, vol. 139, no. 1-4, pp. 3-47, 1996.

[17] T. Belytschko, Y. Y. Lu, and L. Gu, "Element-free Galerkin methods," International Journal for Numerical Methods in Engineering, vol. 37, no. 2, pp. 229-256, 1994.

[18] Y. Cheng, F. Bai, C. Liu, and M. Peng, "Analyzing nonlinear large deformation with an improved element-free Galerkin method via the interpolating moving least-squares method," International Journal of Computational Materials Science and Engineering, vol. 5, no. 4, Article ID 1650023, 2016.

[19] Y. M. Cheng, F. N. Bai, and M. J. Peng, "A novel interpolating element-free Galerkin (IEFG) method for two-dimensional elastoplasticity," Applied Mathematical Modelling, vol. 38, no. 2122, pp. 5187-5197, 2014.

[20] T. Belytschko, Y. Y. Lu, and L. Gu, "Crack propagation by element-free Galerkin methods," Engineering Fracture Mechanics, vol. 51, no. 2, pp. 295-315, 1995.

[21] R. Das and P. W. Cleary, "A mesh-free approach for fracture modelling of gravity dams under earthquake," International Journal of Fracture, vol. 179, no. 1-2, pp. 9-33, 2013.

[22] Y.-M. Cheng, C. Liu, F.-N. Bai, and M.-J. Peng, "Analysis of elastoplasticity problems using an improved complex variable element-free Galerkin method," Chinese Physics B, vol. 24, no. 10, Article ID 100202, 2015.

[23] J. Dolbow, N. Moes, and T. Belytschko, "An extended finite element method for modeling crack growth with frictional contact," Computer Methods in Applied Mechanics and Engineering, vol. 190, no. 51-52, pp. 6825-6846, 2001.

[24] A. Afshar, A. Daneshyar, and S. Mohammadi, "XFEM analysis of fiber bridging in mixed-mode crack propagation in composites," Composite Structures, vol. 125, pp. 314-327, 2015.

[25] F. Liao and Z. Huang, "An extended finite element model for modelling localised fracture of reinforced concrete beams in fire," Computers \& Structures, vol. 152, pp. 11-26, 2015.

[26] V. S. Kothnur, S. Mukherjee, and Y. X. Mukherjee, "Two-dimensional linear elasticity by the boundary node method," International Journal of Solids and Structures, vol. 36, no. 8, pp. 11291147, 1998.

[27] F. Yan, X.-T. Feng, J.-H. Lv, P.-Z. Pan, and S.-J. Li, "A new dual reciprocity hybrid boundary node method based on Shepard and Taylor interpolation method and Chebyshev polynomials," Engineering Analysis with Boundary Elements, vol. 73, pp. 61-68, 2016.
[28] K. M. Liew, Y. Cheng, and S. Kitipornchai, "Boundary elementfree method (BEFM) and its application to two-dimensional elasticity problems," International Journal for Numerical Methods in Engineering, vol. 65, no. 8, pp. 1310-1332, 2006.

[29] B. D. Dai and Y. M. Cheng, "An improved local boundary integral equation method for two-dimensional potential problems," International Journal of Applied Mechanics, vol. 2, no. 2, pp. 421436, 2010.

[30] M. Peng and Y. Cheng, "A boundary element-free method (BEFM) for two-dimensional potential problems," Engineering Analysis with Boundary Elements, vol. 33, no. 1, pp. 77-82, 2009.

[31] Y. Sun, B. Chen, Y. Zhu, and Z. Zhang, "Higher-order continuum model and mesh-free simulation for microtubules under hydrostatic presssure," Nanoscience and Nanotechnology Letters, vol. 4, no. 6, pp. 593-597, 2012.

[32] Y. Sun and K. M. Liew, "The buckling of single-walled carbon nanotubes upon bending: The higher order gradient continuum and mesh-free method," Computer Methods in Applied Mechanics and Engineering, vol. 197, no. 33-40, pp. 3001-3013, 2008.

[33] Z. Tang, S. Shen, and S. N. Atluri, "Analysis of materials with strain-gradient effects: a meshless local Petrov-Galerkin (MLPG) approach, with nodal displacements only," Computer Modeling in Engineering and Sciences, vol. 4, no. 1, pp. 177-196, 2003.

[34] G. Monegato, "The numerical evaluation of one-dimensional Cauchy principal value integrals," Computing, vol. 29, no. 4, pp. 337-354, 1982.

[35] Y. Murakanal, Stress Intensity Factors Handbook, vol. 1-2, Pergamon Press, Oxford, UK, 1987.

[36] C. A. Brebbia, The Boundary Element Method for Engineers, Pentech Press, London, UK, 2nd edition, 1984.

[37] P. K. Banerjee and R. Butterfield, Boundary Element Methods in Engineering Science, McGraw-Hill, London, UK, 1981.

[38] K. M. Liew, Y. Sun, and S. Kitipornchai, "Boundary element-free method for fracture analysis of 2-D anisotropic piezoelectric solids," International Journal for Numerical Methods in Engineering, vol. 69, no. 4, pp. 729-749, 2007. 


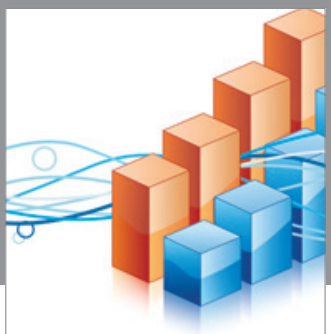

Advances in

Operations Research

vatem alat4

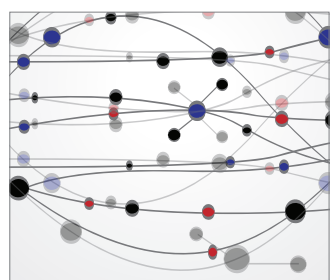

\section{The Scientific} World Journal
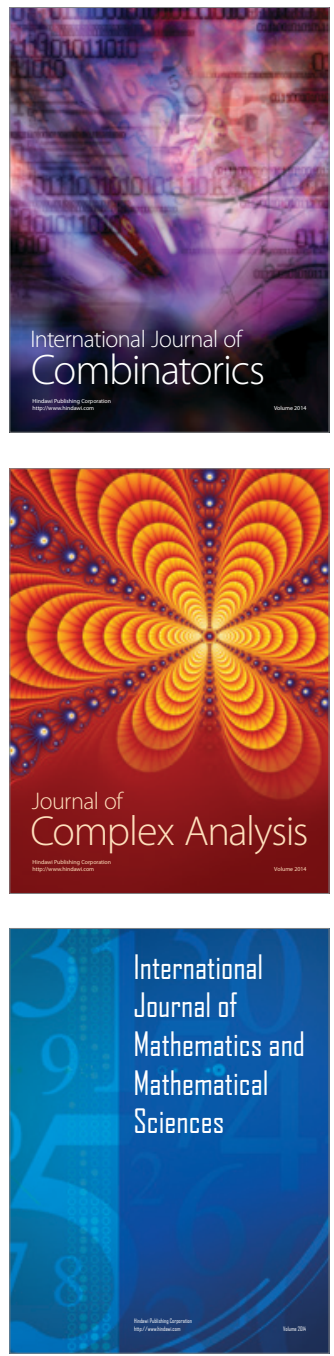
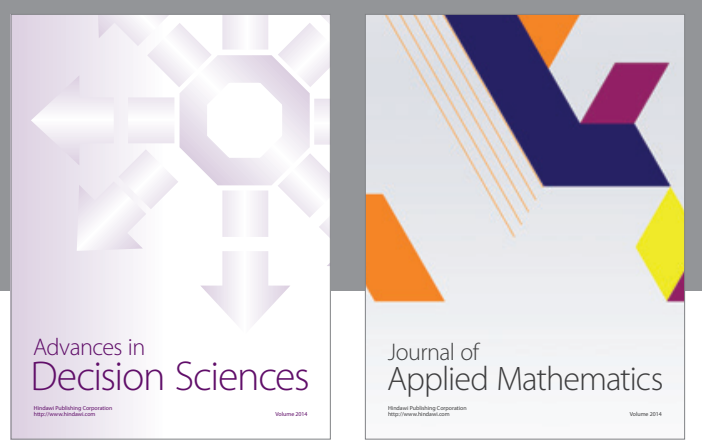

Algebra

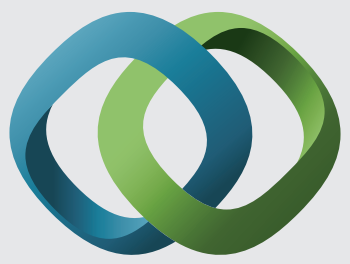

\section{Hindawi}

Submit your manuscripts at

https://www.hindawi.com
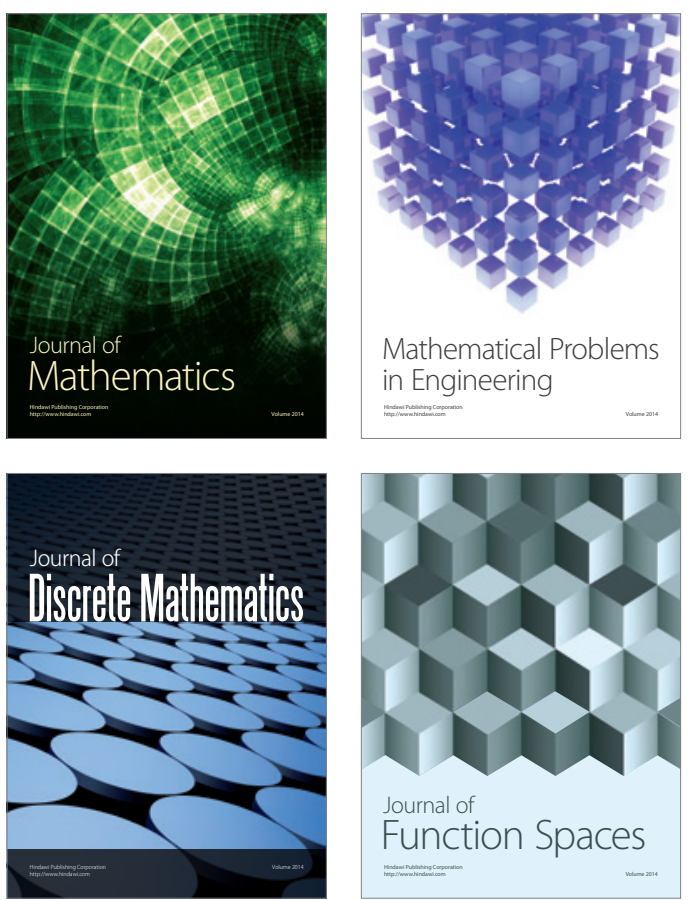

Mathematical Problems in Engineering
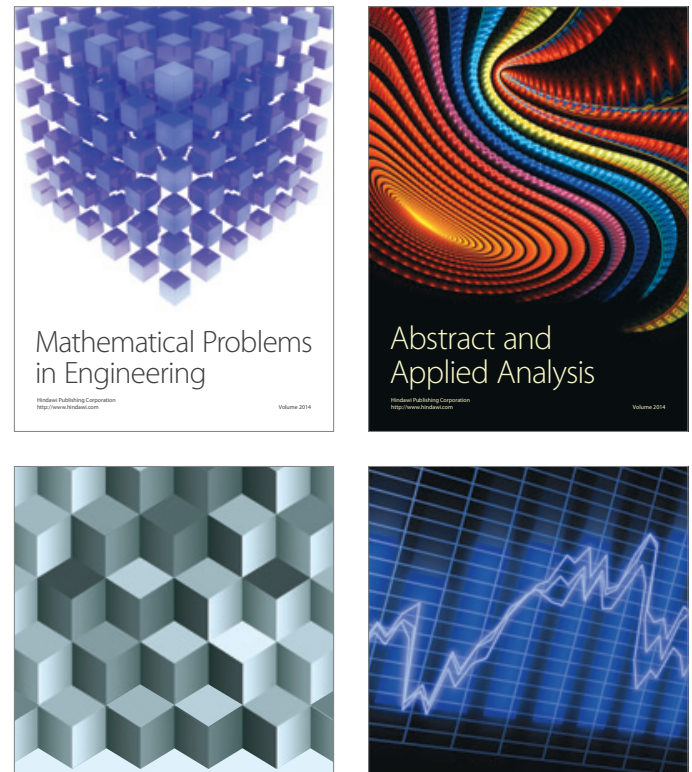

Journal of

Function Spaces

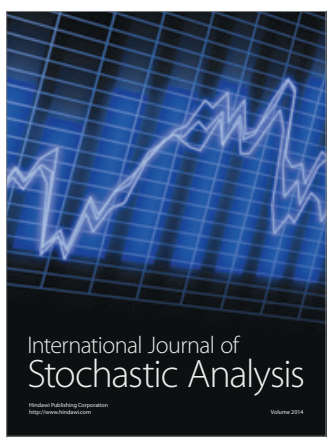

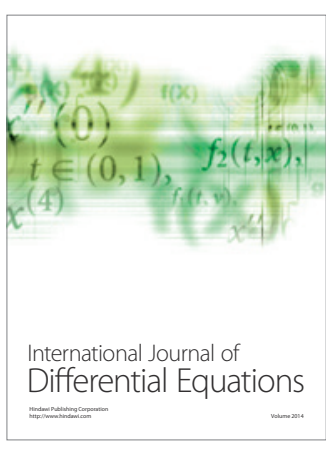
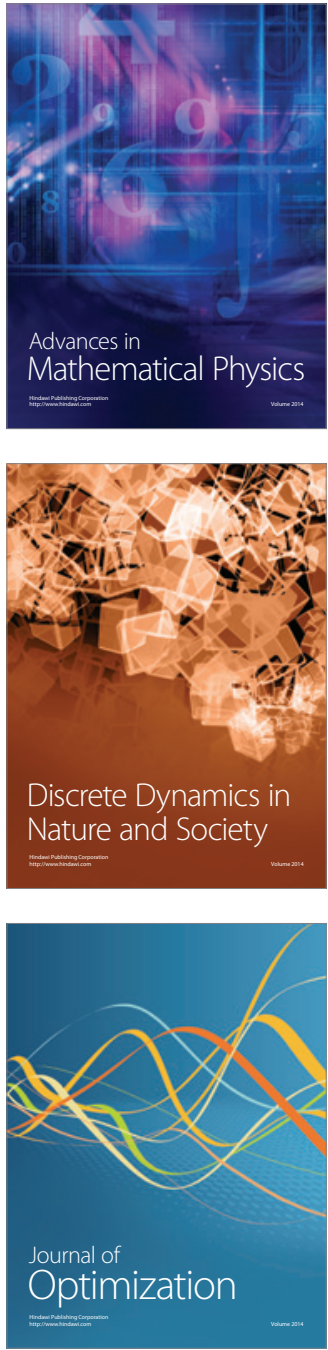